\title{
Ingreso a la universidad y su efecto en el peso de los estudiantes
} University admission and its effect on student weight

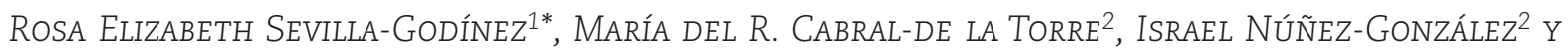
Alicia AlmanZar-CuRieL ${ }^{1}$

${ }^{1}$ Departamento de Ciencias Sociales; ${ }^{2}$ Departamento de Enfermería para la Atención, Desarrollo y Preservación de la Salud Comunitaria. Universidad de Guadalajara, Centro Universitario de Ciencias de la Salud, Guadalajara, México

\section{RESUMEN}

Introducción: El 37\% de la población mundial tiene sobrepeso y obesidad. En México la prevalencia de estas condiciones ha aumentado especialmente en el grupo de edad de 12 a 24 años. La época de ingreso a la Universidad ha sido señalado como una etapa de riesgo para obesidad. Material y métodos: Estudio observacional longitudinal realizado en estudiantes del Centro Universitario de Ciencias de la Salud (CUCS) a su ingreso y un año más tarde. Las variables nivel académico, edad, sexo, peso, talla y el índice de masa corporal (IMC) fueron capturadas en un registro de salud. Las medidas antropométricas las recogió un equipo multidisciplinario de la Unidad de Atención Primaria para la Salud Universitaria. Resultados: Se incluyeron 782 (62\%) estudiantes de diferentes licenciaturas y carreras técnicas del CUCS. Médico cirujano y partero, así como Enfermería, fueron las licenciaturas con mayor número de estudiantes con sobrepeso. Existieron diferencias significativas en el IMC basal y al año para hombres y mujeres, así como por carreras. Discusión: Las mujeres universitarias presentaron menor prevalencia de obesidad y sobrepeso. El ingreso a la universidad genera un incremento en el sobrepeso y obesidad. La universidad debe proporcionar formación en higiene alimentaria a sus futuros profesionales.

Palabras clave: Universidades. Sobrepeso. Obesidad. Estudiantes. Alimento. Índice de masa corporal.

\begin{abstract}
Introduction: $37 \%$ of the world population is overweight and obese. In Mexico, the prevalence of those conditions have increased, especially in the age group of 12 to 24 years. Admission to the University has been indicated as a risk stage for obesity. Material and methods: Longitudinal observational study carried out in CUCS (Centro Universitario de Ciencias de la Salud) students on admission and one year later. The variables of academic degree, age, gender, weight, height and body mass index (BMI) were captured in a health record. The anthropometric measures were collected by a multidisciplinary team from the Primary Health Care Unit. Results: 782 (62\%) students from different degrees and technical careers from CUCS were included. Surgeon and Obstetrician as well as Nursing were the degrees with the highest number of overweight students. There were significant differences in the baseline BMI and one year later BMI for men and women, as well as among careers. Discussion: University women had lower prevalence of overweight and obesity. The entrance to the university generates an increase in overweight and obesity. The university must provide training in food hygiene to its future professionals.
\end{abstract}

Key words: Universities. Overweight. Obesity. Students. Food. Body mass index.

\section{Correspondencia:}

*Rosa Elizabeth Sevilla-Godínez

E-mail: elizros@hotmail.com
Fecha de recepción: 26-02-2018

Fecha de aceptación: 25-10-2019

DOI: 10.24875/RME.19001825
Disponible en internet: 28-11-2019 Rev Mex Endocrinol Metab Nutr. 2019;6:193-9

2462-4144/@ 2019 Sociedad Mexicana de Nutricion y Endocrinologia, AC. Publicado por Permanyer México. Este es un artículo Open Access bajo la licencia CC BY-NC-ND (http://creativecommons.org/licenses/by-nc-nd/4.0/). 


\section{INTRODUCCIÓN}

La obesidad es un problema mundial de salud pública. Se caracteriza por una ingesta mayor de calorías a las necesarias ${ }^{1}$. Uno de los índices más aceptados para medir el sobrepeso y obesidad es el índice de masa corporal (IMC), que correlaciona dos parámetros, la estatura y el peso. De acuerdo con la Norma Oficial Mexicana ${ }^{2}$ la obesidad se define como «...enfermedad caracterizada por el exceso de tejido adiposo en el organismo, la cual se determina cuando en las personas adultas existe un IMC igual o mayor a $30 \mathrm{~kg} / \mathrm{m}^{2} \ldots .$. , mientras que el sobrepeso se ubica entre 25 y $29.9 \mathrm{~kg} / \mathrm{m}^{2}$. Los intervalos del IMC también incluyen desnutrición $\left(<18.5 \mathrm{~kg} / \mathrm{m}^{2}\right)$ y peso normal $\left(18.5 \text { a } 24.9 \mathrm{~kg} / \mathrm{m}^{2}\right)^{3}$.

El 37\% de la población adulta mundial tiene sobrepeso y obesidad ${ }^{4}$. En México ${ }^{3}$ el porcentaje de adolescentes (12 y 19 años) con sobrepeso y obesidad aumentó un $3 \%$ en un espacio de 6 años. En el grupo de 20 a 24 años se ha incrementado en ambos sexos en un $14 \%$, y no es sino hasta el grupo de edad de 65 a 69 años que comienza a descender. Además, se ha estimado que una de cada tres personas tiene un problema de salud relacionado con la obesidad ${ }^{5}$. Destaca como una de las causas principales de motivos de consulta ${ }^{3}$ y se considera precursora para desarrollar problemas de hipercolesterolemia, hipertensión, diabetes mellitus o asma ${ }^{6,7}$, lo que contribuye al desarrollo de las enfermedades cardiovasculares, que son la causa número uno de mortalidad mundial ${ }^{8}$, y en México son responsables del $32 \%$ de las muertes en mujeres y el $20 \%$ en hombres ${ }^{9}$.

Un grupo importante de atención son los universitarios, ya que de acuerdo con las estadísticas una tercera parte de ellos es obesa. Es alarmante si se aborda desde un punto de vista epidemiológico, puesto que la población entre 20 y 24 años de edad, que representa el $8.6 \%$ de la población total ${ }^{10} \mathrm{y}$ en un lapso menor de 10 años formará parte de la población económicamente activa ${ }^{11}$, se encuentra en riesgo de desarrollar enfermedades debido a la obesidad, con un elevado costo empresarial y social asociado. Situación compleja si consideramos que en las siguientes tres décadas habrá una concentración mayor de la población en el grupo de edad entre 15 y 65 años, quienes representan el «bono demográfico» ${ }^{12}$ que permitiría invertir en capital humano y favorecer el desarrollo del país. A su vez, en esos 10 años se proyecta ${ }^{13}$ un descenso en la proporción de población adulta mexicana con peso normal y un incremento en sobrepeso y obesidad a partir del año 2020 al 2050. Desde un enfoque económico, el sobrepeso incrementa hasta un $36 \%$ los costos de atención médica ${ }^{14}$ y un $10 \%$ los costos de prescripción ${ }^{6}$, sin considerar los costos debidos a una mayor discapacidad, baja productividad, absentismo, muerte prematura y disminución de la calidad de vida 6 . Un programa de prevención para tratar la obesidad implicaría un costo de \$12 USD per cápita en México y evitaría 55,000 muertes cada año ${ }^{15}$. Por otro lado, desde el punto de vista cultural y social, la educación y hábitos nocivos en salud que transmita esta generación a sus hijos simularán un efecto de boomerang donde el daño se transmitirá generación tras generación cada vez con mayor gravedad. Por tanto, es un reto para las universidades atender a esta población. Sin embargo, la etapa universitaria ha sido considerada ${ }^{1,16}$ de alto riesgo para aumentar de peso. En la universidad los jóvenes cambian sus patrones de alimentación, incrementan el consumo de productos ricos en grasas, azúcares y refrescos, y disminuyen el de frutas y verduras ${ }^{17}$, disminuyen el ejercicio físico y aumentan las horas sedentarias ${ }^{1,16}$. Además sufren cambios emocionales, como depresión, ansiedad, baja autoestima y soledad', que llevan a comer de manera desmesurada. Es decir, una persona ante situaciones de ansiedad puede tender a consumir alimentos para mitigar esta angustia.

El propósito de esta investigación fue analizar longitudinalmente el IMC de los estudiantes a su ingreso a la universidad para evaluar algún cambio posterior al primer semestre de vida universitaria.

\section{MATERIAL Y MÉTODOS}

Estudio observacional longitudinal realizado en estudiantes del Centro Universitario de Ciencias de la Salud (CUCS) con medición de las variables de interés en dos momentos diferentes. El estudio abarcó un lapso de un año, en él se incluyeron 787 estudiantes (62\%) de primer ingreso de las licenciaturas de 
Tabla 1. Frecuencia de alumnos y promedio de edad para hombres y mujeres por licenciatura de estudio

\begin{tabular}{|c|c|c|c|c|c|}
\hline \multirow[t]{2}{*}{ Licenciatura } & \multirow{2}{*}{$\begin{array}{c}\mathrm{N} .^{\circ} \\
\text { estudiantes } \\
(\%)\end{array}$} & \multicolumn{2}{|c|}{ Porcentaje sexo } & \multirow{2}{*}{$\begin{array}{c}\text { Promedio de } \\
\text { edad } \\
\text { (años) }\end{array}$} & \multirow{2}{*}{$\begin{array}{l}\text { Rango } \\
\text { (años) }\end{array}$} \\
\hline & & Mujer & Hombre & & \\
\hline Cultura física y deporte & $23(2.9)$ & 1.9 & 5.0 & 20.7 & $18-37$ \\
\hline Enfermería técnica & $117(14.8)$ & 16.3 & 11.9 & 20.2 & $15-40$ \\
\hline Licenciatura en enfermería & $153(19.4)$ & 21.7 & 14.9 & 19.5 & $18-34$ \\
\hline Médico cirujano y partero & $219(27.8)$ & 25.7 & 32.2 & 18.6 & $17-28$ \\
\hline Nutrición & $80(10.1)$ & 12.4 & 5.7 & 19.7 & $18-52$ \\
\hline Odontología & $71(9.0)$ & 8.4 & 10.3 & 18.9 & $18-24$ \\
\hline Psicología & $74(9.4)$ & 8.6 & 11.1 & 19.8 & $18-45$ \\
\hline Prótesis dental & $2(0.2)$ & 0.4 & 0.0 & 20.5 & $20-21$ \\
\hline Radiología & $4(0.5)$ & 0.2 & 1.1 & 24.2 & $18-30$ \\
\hline Técnico superior en emergencias médicas & $27(3.4)$ & 1.7 & 6.9 & 20.3 & $18-29$ \\
\hline Terapia física & $17(2.1)$ & 2.9 & 0.8 & 20.4 & $18-30$ \\
\hline Total & $787(100)$ & 100 & 100 & 20.5 & $15-45$ \\
\hline
\end{tabular}

Médico cirujano y partero, Enfermería, Cultura física y deporte, Nutrición, Cirujano dentista, Psicología, así como las carreras técnicas superiores universitarias en Radiología e imagen, Emergencias, seguridad laboral y rescate, Prótesis dental, Terapia física y Enfermería.

Los estudiantes fueron recibidos en la Unidad de Atención Primaria para la Salud Universitaria (UAPSU). Ingresaron en el ciclo 2013 A y durante el mes de enero a junio se les calculó el IMC tras recabar los parámetros de peso y talla. Al término del primer año, en el ciclo escolar $2014 \mathrm{~A}$, durante el mes de enero a junio se repitió el proceso. Estos datos, entre otros, fueron capturados como parte de un registro de salud que se tiene de cada estudiante que ingresa y el cual cada estudiante acepta. Las variables analizadas fueron licenciatura, edad, sexo, peso, talla e IMC.

Las medidas antropométricas las realizó el equipo multidisciplinario compuesto por pasantes de la carrera de enfermería, de las licenciaturas en Enfermería, Médico cirujano y partero, Cultura física y deportes, Psicología y Nutrición, quienes fueron capacitados en todo momento por un doctor en salud pública y por pasantes de nutrición en un taller de medidas. Se utilizó una báscula calibrada previamente antes de cada utilización.

El muestreo fue a conveniencia y se trató de incluir al $100 \%$ de los estudiantes. Sin embargo, no todos asistieron, solo se logró integrar al $62 \%$. Se procedió a identificar en la base de datos los estudiantes del ciclo escolar 2013; se revisó su horario de clases a fin de poder localizarlos en un momento y lugar determinado; se solicitó permiso a los profesores para que los dejaran acudir en ese momento y proceder a tomar medidas y peso. Una vez en la UAPSU se registró su asistencia en la base de datos con un color en su primera visita y otro color en la segunda visita; se registraron las medidas y se entregó cartilla de vacunación. Se excluyeron aquellas alumnas que estuvieran embarazadas, y en general a quienes desertaron o no acudieron; así mismo en la segunda toma se eliminó aquello que mostraba una diferencia importante en cuanto a los datos de la primera visita, es decir, que sugiriera error en la medición de las variables estudiadas.

El procesamiento y análisis de los datos fue realizado en los programas Microsoft Excel $^{\circledR} 2016$ e IBM SPSS ${ }^{\circledR}$ para Windows, versión 22.0.

Se realizó estadística descriptiva para estimar medidas de frecuencia, medias y desviaciones estándar. EI IMC entre grupos independientes (hombres y mujeres) se comparó mediante la prueba t de Student a la basal y al año. Así también, se realizó la comparación del IMC entre grupos pareados (a la basal vs. 1 año) mediante la prueba t para datos pareados. Adicionalmente se estimaron intervalos de confianza al 95\% para diferencias de medias. Un valor de alfa menor que 0.05 fue utilizado para fijar el nivel de significancia. 
Tabla 2. Comparación del IMC de acuerdo con el sexo: a la basal y al año

\begin{tabular}{lccc}
\hline & Mujer & Hombre & $\mathrm{p}^{*}$ \\
\hline IMC basal & $23.2 \pm 0.21$ & $24.3 \pm 0.28$ & $<0.001$ \\
\hline IMC a los 6 meses & $23.6 \pm 0.21$ & $24.8 \pm 0.27$ & $<0.001$ \\
\hline & Cambio de IMC basal y al año & $\mathrm{p}^{\dagger}$ \\
\hline Mujeres & & $0.42 \pm 0.11$ & $<0.001$ \\
\hline Hombres & $0.50 \pm 0.14$ & $<0.001$ \\
\hline
\end{tabular}

Resultados expresados como medias \pm desviaciones estándar.

*Comparación entre grupos independientes (mujeres vs. hombres) mediante la prueba t de Student.

${ }^{\dagger}$ Comparación entre grupos pareados (IMC basal vs. IMC al año) mediante la prueba t para grupos pareados.

IMC: índice de masa corporal.

\section{RESULTADOS}

Los alumnos dictaminados en el ciclo 2013 A fueron 1,269 , y en el estudio se integraron un total de 787 (62\%) estudiantes de las diferentes licenciaturas y carreras técnicas (Tabla 1). El mayor porcentaje de alumnos se concentró en la licenciatura de Médico cirujano y partero (28\%). En general las mujeres representaron el $67 \%$, con una razón mujer:hombre de 2:1. Solamente en las carreras técnicas superiores universitarias de Radiología e imagen y de Emergencias, seguridad laboral y rescate, predominaron los hombres, con una razón 3:1 y 2:1 respectivamente. La mayoría de los estudiantes (69\%) tenían entre 18 y 19 años. El promedio global de edad fue 19.5 años. Fue mayor la proporción de mujeres (31.5\%) que se encontraban entre sobrepeso y obesidad que la de los hombres (19.8\%).

Los estudiantes con mayor sobrepeso tanto en el primero como segundo semestre fueron de las licenciatura de Médico cirujano y partero y de Enfermería, con un 31 y un $21 \%$ respectivamente.

De manera global, el $59.5 \%$ de los estudiantes se encontraba en peso normal en el primer semestre, lo que disminuyó a un $58.3 \%$ en el segundo semestre $(p=0.68)$, el $20.8 \%$ se encontraba en sobrepeso y pasó al $23.5 \%$ en el segundo semestre $(p=0.90)$, y el $9.5 \%$ estaba en obesidad y aumentó al $11.0 \%$ en el segundo semestre $(p=0.83)$.

Del total de los estudiantes que estaban en IMC normal a su ingreso universitario, el $12.8 \%$ pasaron a sobrepeso y el $1.1 \%$ a obesidad después de un año. En la tabla 2 se muestra una diferencia estadísticamente significativa en el cambio del parámetro de IMC basal y posterior a un año para hombres y mujeres en general y también al interior, tanto en el grupo de mujeres como en el de hombres.

Existió un cambio significativo desde el IMC basal al IMC posterior a un año según la carrera (en general), sin separar hombres y mujeres analizados con prueba t para grupos pareados o relacionados (antes y después), en las licenciaturas de Odontología, Medicina, Psicología y licenciatura en Enfermería, así como en la carrera técnica de Enfermería (Tabla 3).

\section{DISCUSIÓN}

En nuestro estudio encontramos a un poco más de la mitad de los estudiantes con peso normal, y cerca del $35 \%$ con sobrepeso y obesidad, lo que coincide con otras universidades ${ }^{14,18}$ y nos coloca por encima de lo estimado en universitarios latinoamericanos ${ }^{19,20}$.

Similar $^{8,14,18}$ y contrario ${ }^{7}$ a lo referido en otras universidades, encontramos menor sobrepeso en las mujeres en ambas mediciones. Esto resulta relevante al considerar que son las mujeres quienes culturalmente están a cargo de una familia, educan y forman en valores y hábitos. De ahí la importancia de formar una higiene alimentaria desde la universidad para que pueda trascender a generaciones posteriores. Esto puede generar un impacto positivo porque siendo la madre quien regularmente cocina 
Tabla 3. Basal y comparativa de IMC de las licenciaturas del Centro Universitario de Ciencias de la Salud, en general y según el sexo

\begin{tabular}{|c|c|c|c|c|c|}
\hline & $\mathrm{n}$ & $\begin{array}{c}\text { IMC } 1 \\
\text { Media } \pm \text { DE }\end{array}$ & $\begin{array}{c}\text { IMC } 2 \\
\text { Media } \pm \text { DE }\end{array}$ & Diferencia (IC 95\%) & $\mathrm{p}^{*}$ \\
\hline \multicolumn{6}{|c|}{ Cultura física y deporte } \\
\hline General & 23 & $23.98 \pm 3.3$ & $23.59 \pm 3.0$ & $-0.39(-0.98$ a 0.19$)$ & 0.184 \\
\hline Hombres & 13 & $24.03 \pm 3.3$ & $23.81 \pm 2.8$ & $-0.22(-0.93$ a 0.48$)$ & 0.503 \\
\hline Mujeres & 10 & $23.92 \pm 1.1$ & $23.31 \pm 1.0$ & $-0.60(-1.76$ a 0.55$)$ & 0.267 \\
\hline \multicolumn{6}{|c|}{ Enfermería técnica } \\
\hline General & 117 & $24.25 \pm 5.3$ & $24.99 \pm 5.4$ & $0.74(0.41$ a 1.06$)$ & $<0.001$ \\
\hline Hombres & 31 & $25.74 \pm 5.0$ & $26.11 \pm 4.6$ & $0.37(-0.37$ a 1.12$)$ & 0.311 \\
\hline Mujeres & 86 & $23.72 \pm 0.5$ & $24.59 \pm 0.6$ & $0.86(0.49$ a 1.23$)$ & 0.000 \\
\hline \multicolumn{6}{|c|}{ Licenciatura en enfermería } \\
\hline General & 153 & $24.07 \pm 5.3$ & $24.51 \pm 5.3$ & $0.43(0.01$ a 0.56$)$ & 0.042 \\
\hline Hombres & 39 & $24.70 \pm 4.6$ & $25.21 \pm 4.9$ & $0.50(-0.09$ a 1.10$)$ & 0.096 \\
\hline Mujeres & 114 & $23.74 \pm 0.5$ & $24.18 \pm 0.5$ & $0.43(-0.09$ a 0.97$)$ & 0.108 \\
\hline \multicolumn{6}{|c|}{ Médico cirujano y partero } \\
\hline General & 219 & $23.41 \pm 4.1$ & $23.77 \pm 4.3$ & 0.36 (0.07 a 0.64) & 0.012 \\
\hline Hombres & 84 & $24.51 \pm 4.1$ & $25.05 \pm 4.0$ & 0.53 (0.04 a 1.02) & 0.032 \\
\hline Mujeres & 135 & $22.76 \pm 0.3$ & $23.03 \pm 0.3$ & $0.26(-0.09$ a 0.62$)$ & 0.150 \\
\hline \multicolumn{6}{|l|}{ Nutrición } \\
\hline General & 80 & $23.06 \pm 4.2$ & $22.85 \pm 4.1$ & $-0.20(-0.62$ a 0.20$)$ & 0.321 \\
\hline Hombres & 15 & $23.59 \pm 4.5$ & $23.18 \pm 4.0$ & $-0.41(-1.40$ a 0.57$)$ & 0.386 \\
\hline Mujeres & 65 & $22.99 \pm 0.5$ & $22.81 \pm 0.5$ & $-0.18(-0.65$ a 0.28$)$ & 0.441 \\
\hline \multicolumn{6}{|l|}{ Odontología } \\
\hline General & 71 & $22.33 \pm 3.8$ & $22.94 \pm 3.2$ & 0.61 (0.18 a 1.03) & 0.006 \\
\hline Hombres & 27 & $23.23 \pm 4.4$ & $23.70 \pm 3.6$ & $0.47(-0.35$ a 1.29$)$ & 0.25 \\
\hline Mujeres & 44 & $21.78 \pm 0.4$ & $22.48 \pm 0.4$ & 0.69 (0.20 a 1.19) & 0.007 \\
\hline \multicolumn{6}{|l|}{ Psicología } \\
\hline General & 74 & $23.38 \pm 4.7$ & $24.70 \pm 5.1$ & $1.31(0.27$ a 2.36$)$ & 0.014 \\
\hline Hombres & 29 & $23.11 \pm 4.7$ & $24.87 \pm 5.3$ & $1.76(0.30$ a 3.22$)$ & 0.019 \\
\hline Mujeres & 45 & $23.55 \pm 0.7$ & $24.58 \pm 0.7$ & $1.03(-0.43$ a 2.50$)$ & 0.164 \\
\hline \multicolumn{6}{|c|}{ Prótesis dental } \\
\hline General & 2 & $24.36 \pm 3.8$ & $23.37 \pm 1.5$ & $-0.99(-21.69$ a 19.71) & 0.652 \\
\hline Hombres & 0 & - & - & - & - \\
\hline Mujeres & 2 & $24.36 \pm 2.6$ & $23.37 \pm 1.0$ & $-0.99(-21.69$ a 19.71$)$ & 0.652 \\
\hline \multicolumn{6}{|l|}{ Radiología } \\
\hline General & 4 & $24.57 \pm 3.7$ & $24.33 \pm 2.7$ & $-0.23(-2.20$ a 1.73$)$ & 0.730 \\
\hline Hombres & 3 & $25.98 \pm 2.9$ & $25.25 \pm 2.5$ & $-0.73(-2.98$ a 1.52$)$ & 0.298 \\
\hline Mujeres & 1 & 20.34 & 21.59 & - & - \\
\hline \multicolumn{6}{|c|}{$\begin{array}{l}\text { Técnico superior en } \\
\text { emergencias médicas }\end{array}$} \\
\hline General & 27 & $23.65 \pm 5.1$ & $23.88 \pm 4.5$ & $0.23(-0.40$ a 0.86$)$ & 0.461 \\
\hline Hombres & 18 & $23.86 \pm 4.9$ & $23.96 \pm 4.3$ & $0.10(-0.79$ a 1.00$)$ & 0.813 \\
\hline Mujeres & 9 & $23.22 \pm 1.9$ & $23.71 \pm 1.7$ & $0.48(-0.38$ a 1.36$)$ & 0.234 \\
\hline \multicolumn{6}{|l|}{ Terapia física } \\
\hline General & 17 & $23.21 \pm 4.2$ & $23.05 \pm 4.3$ & $-0.15(-0.73$ a 0.41$)$ & 0.573 \\
\hline Hombres & 2 & $22.46 \pm 0.7$ & $22.90 \pm 1.4$ & $0.43(-5.80$ a 6.67$)$ & 0.539 \\
\hline Mujeres & 15 & $23.31 \pm 1.1$ & $23.07 \pm 1.1$ & $-0.23(-0.87$ a 0.40$)$ & 0.445 \\
\hline
\end{tabular}


y enseña la tradición alimentaria a los hijos, estos pueden adquirir hábitos saludables.

En los hombres encontramos una similitud con otros estudios ${ }^{7,18}$ en el momento del ingreso a la universidad, al mostrar casi una tercera parte sobrepeso y una décima parte obesidad.

Cabe destacar que las licenciaturas de Nutrición y de Cultura física y deportes mostraron una disminución en el IMC posterior al ingreso a la universidad. Es importante destacar este aspecto, considerando que son áreas donde se adquieren conocimientos de la alimentación y donde se puede generar una mayor activación física, respectivamente, razones por las cuales pudo existir una disminución en vez de un incremento.

Por otro lado, al igual que otras universidades $8,11,14$ encontramos un incremento en el IMC, en general, tanto en hombres como en mujeres, una vez que ingresan a la universidad. Esto puede ser explicado porque en esta etapa existen factores angustiantes como la adaptación al estilo de vida universitaria, exigencias académicas, traslado a otra ciudad, dejar amigos y organizar los tiempos de estudio. Sin embargo, también es un área de oportunidad para las universidades que además del compromiso social y académico, deben contribuir a fomentar hábitos sanos mediante acciones extracurriculares y multidisciplinarias que se adapten a las necesidades de los universitarios. Es decir, si se identifica el ingreso a la universidad como un factor que detona el incremento del IMC, se pueden anticipar medidas de prevención y promoción de la salud que permitan a los estudiantes afrontar de manera adecuada este proceso de adaptación en sus hábitos alimenticios. Y no solo con el objetivo de disminuir el sobrepeso y obesidad, sino también para formar seres humanos competentes, productivos y sanos; individuos con capital salud que les permita desarrollar sus actividades y con esto contribuir al desarrollo social, económico y cultural del país. Incluso asumir la responsabilidad de cuidarse y transferir su conocimiento con sus pacientes en el área donde se desenvuelvan como futuros profesionales. Además esto puede lograr paulatinamente una disminución de las primeras causas de morbilidad que tienen como común denominador una alimentación inadecuada. Ya lo señalaba Hipócrates «que tu alimento sea tu medicina».
FUENTES DE FINANCIAMIENTO

La presente investigación no ha recibido ayudas específicas provenientes de agencias del sector público, sector comercial o entidades sin ánimo de lucro.

\section{BIBLIOGRAFÍA}

1. Garay SG. Sobrepeso y obesidad en el universitario: Implicaciones en la Consejería. Griot. 2011;4(1):25-34.

2. Secretaría de Salud. Norma Oficial Mexicana NOM-008-SSA3-2010, Para el tratamiento integral del sobrepeso y obesidad. Diario Oficial de la Federación, 4 de agosto de 2010.

3. Barquera S, Campos NI, Hernández BL, Pedroza TA, Rivera JA. Prevalencia de obesidad en adultos mexicanos, ENSANUT 2012. Salud pública Méx. 2013;55(supl 2):s151-60.

4. Obesity and overweight increasing worldwide [Internet]. Infographic: GBD 2013 Obesity). IHME Measuring what matters; 28 de mayo de 2014. Disponible en: http://www.healthdata.org/infographic/obesity-andoverweight-increasing-worldwide

5. Padilla SS, Guzmán LA, Baltazar Al, Gallegos LF. Incidencia de sobrepeso y obesidad en una población de alumnos de primer ingreso del Centro Universitario de Ciencias de la Salud durante el Ciclo 2010-B. Rev Med MD. 2011;2(3). 123-126.

6. Hammond RA, Levine R. The economic impact of obesity in the United States. Diabetes Metab Syndr Obes. 2010;3:285-95.

7. Gómez PG, González CM, Sois TC, Cuevas RC, Unikel SC, Saucedo MT, et al. Obesidad en población universitaria: prevalencia y relación con agresión y conductas compensatorias y alimentarias de riesgo. Rev Mex Psicol. 2006;23(2):135-47.

8. Aguilar YA, Pérez LD, Rodríguez GL, Hernández SL, Jiménez GF, Rodríguez GR. Prevalencia estacionaria de sobrepeso y obesidad en universitarios del sur de Veracruz, México. Med Univers. 2010;12(46):24-8.

9. Gobierno de la República. Plan Nacional de Desarrollo 2013-2018. Programa Sectorial de Salud. Diario Oficial de la Federación, 1.a ed. 2014.

10. Instituto Nacional de Estadística y Geografía. México, 11 julio de 2013.

11. Gallardo WI, Buen LL. Mala nutrición en estudiantes universitarios de la Escuela de Dietética y Nutrición del ISSSTE. Rev Med UV. 2011;(1)6-11.

12. La población mexicana asciende a 118.4 millones. El Informador (México). 30 de junio de 2013.

13. Rtveladze K, Marsh T, Barquera S, Sánchez LM, Levy D, Melendez G, et al. Obesity prevalence in Mexico: impact on health and economic burden. Public Health Nutrition. 2013;17(1):233-239.

14. Hidalgo CA, Ramírez LG, Montaño ER, Hidalgo SA. Sobrepeso medido por IMC o percibido y comportamientos de control del peso en adolescentes universitarios de Ciudad Guzmán, Jalisco, México. Archivos Latinoamericanos de Nutrición. 2012;62(1):44-52.

15. Sassi F. Obesity and the economics of prevention. Fit not Fat. Organisation for Economic Co-operation and Development; 2010.

16. Salazar C, Feu S, Vizuete CM, Cruz SE. IMC y actividad física de los estudiantes de la Universidad de Colima. Revista Internacional de Medicina y Ciencias de la Actividad Física y el Deporte. 2013;13(51):569-84.

17. Franco PK, Valdés ME. Frecuencia de consumo de alimentos, índice de masa corporal y porcentaje de grasa en estudiantes universitarios: un estudio longitudinal. Ciencia UAT. 2013;25(1):18-22.

18. Trujillo HB, Vásquez $C$, Almanza JR, Jaramillo ME, Mellin TE, Valle OB, et al. Frecuencia y factores de riesgo asociados a sobrepeso y obesidad en universitarios de Colima, México. Rev Salud Pública. 2010;12(2): 197-207.

19. Inciarte P, González R, Wheeler T, Hernández J, Rojas J, Bermúdez V. Factores de riesgo relacionados con la obesidad en estudiantes universitarios de reciente ingreso de la escuela de medicina de la Universidad del Zulia. Revista Latinoamericana de Hipertensión. 2013;8(2):29-37.

20. Durán AS, Blanco BE, Rodríguez NM, Cordón AK, Salazar AJ, Record CJ, et al. Asociación entre edulcorantes no nutritivos y riesgo de obesidad en estudiantes universitarios de Latinoamérica. Rev Med Chile. 2015;143:367-73. 